Article

\title{
Enhanced Antimicrobial Activity of AamAP1-Lysine, a Novel Synthetic Peptide Analog Derived from the Scorpion Venom Peptide AamAP1
}

\author{
Ammar Almaaytah ${ }^{1{ }^{*}}$, Shadi Tarazi ${ }^{2}$, Ahmad Abu-Alhaijaa ${ }^{2}$, Yara Altall ${ }^{1}$, \\ Nizar Alshar'i ${ }^{3}$, Khaldon Bodoor ${ }^{4}$ and Qosay Al-Balas ${ }^{3}$
}

1 Department of Pharmaceutical Technology, Faculty of Pharmacy, Jordan University of Science and Technology, P.O. Box 3030, Irbid 22110, Jordan; E-Mail: yraltall@just.edu.jo

2 Department of Applied Biological Sciences, Faculty of Science and Arts, Jordan University of Science and Technology, P.O. Box 3030, Irbid 22110, Jordan; E-Mails: shadytarazi89@hotmail.com (S.T.); akaboalhijaa11@sci.just.edu.jo (A.A.-A.)

3 Department of Medicinal Chemistry, Faculty of Pharmacy, Jordan University of Science and Technology, P.O. Box 3030, Irbid 22110, Jordan; E-Mails: nashari@just.edu.jo (N.A.); qabalas@just.edu.jo (Q.A.-B.)

4 Department of Biotechnology and Genetic Engineering, Faculty of Science and Arts, Jordan University of Science and Technology, P.O. Box 3030, Irbid 22110, Jordan; E-Mail: khaldon_bodoor@just.edu.jo

* Author to whom correspondence should be addressed; E-Mail: amalmaaytah@just.edu.jo; Tel.: +962-7-95550234; Fax: +962-2-7201075.

Received: 7 March 2014; in revised form: 9 April 2014 / Accepted: 14 April 2014 / Published: 25 April 2014

\begin{abstract}
There is great interest in the development of antimicrobial peptides as a potentially novel class of antimicrobial agents. Several structural determinants are responsible for the antimicrobial and cytolytic activity of antimicrobial peptides. In our study, a new synthetic peptide analog, AamAP1-Lysine from the naturally occurring scorpion venom antimicrobial peptide AamAP1, was designed by modifying the parent peptide in order to increase the positive charge and optimize other physico-chemical parameters involved in antimicrobial activity. AamAP1-Lysine displayed potent antibacterial activity against Gram-positive and Gram-negative bacteria. The minimum inhibitory concentration was in the range of 5 to $15 \mu \mathrm{M}$ with a 10 fold increase in potency over the parent peptide. The hemolytic and antiproliferative activity of AamAP1-Lysine against eukaryotic mammalian cells was minimal at the concentration range needed to inhibit bacterial growth.
\end{abstract}


The antibacterial mechanism analysis indicated that AamAP1-Lysine is probably inducing bacterial cell death through membrane damage and permeabilization determined by the release of $\beta$-galactosidase enzyme from peptide treated $E$. coli cells. DNA binding studies revealed that AamAP1-Lysine caused complete retardation of DNA migration and could display intracellular activities in addition to the membrane permeabilization mode of action reported earlier. In conclusion, AamAP1-Lysine could prove to be a potential candidate for antimicrobial drug development in future studies.

Keywords: antimicrobial peptides; peptide design; membrane-permeation; scorpion peptide; molecular modeling

\section{Introduction}

Antimicrobial resistance currently represents one of the biggest challenges facing human health and the medical community. The misuse of antibiotics over the past decades has resulted in the emergence of several multi-drug resistant strains of microorganisms [1-3]. This problem has created an urgent need to develop novel classes of antimicrobial agents with different modes of action than the conventionally used antibiotics to control this problem [4,5]. Unfortunately, the number of antibiotics being developed and reaching the clinic in the recent years has declined significantly [6]. This decline is mainly attributed to the lack of investment by pharmaceutical companies in this field as their research attention has shifted in recent years towards more financially feasible and profitable areas of drug development [7]. One of the promising alternatives for conventional antibiotics is antimicrobial peptides (AMPs) as this group of molecules display potent activities against target cells, rapid killing kinetics and a broad spectrum of activity against different microbial strains [8-11]. Additionally, these peptides are usually gene coded in host organisms and are expressed either constitutively or induced by various external stimuli making the selection of resistant mutants to AMPs in vitro relatively difficult $[10,11]$.

AMPs represent the first line of innate immune defense mechanism against infectious agents of a variety of organisms including insects, invertebrates, plants, birds and mammals [12,13]. Most AMPs share several common features, including a relatively small size (12-50 amino acid residues long), cationic nature (a net positive charge ranging from +2 to +9 ) and an amphipathic structure containing approximately 50\% hydrophobic residues [14,15]. Among the various AMPs discovered, cationic $\alpha$-helical peptides represent the most abundant and well studied group of AMPs [16]. Cationic $\alpha$-helical AMPs are known to unlikely evoke bacterial resistance due to their ability to target bacterial membranes through electrostatic interactions and to bind lipid components of bacterial membranes. One of the major issues that have delayed the development of cationic AMPs is the ability of the peptides to cause significant damage to mammalian membranes and the lack of selectivity to distinguish microbial cells from mammalian cells on the basis of their different lipid membrane components. Thus, cationic AMPs displaying high selectivity towards microbial cells and low toxicity against mammalian cells and human erythrocytes would represent attractive candidates for use as therapeutic agents $[17,18]$. 
In pursuit of novel antimicrobial agents, several peptides from scorpion venoms with antimicrobial activity were identified recently [19-22]. The first scorpion AMP was Hadrurin from the venom of the scorpion Hadrurus aztecus [23], this discovery was followed by literature reports of several other scorpion AMPs. Recently, a novel AMP from the venom of the North African scorpion Androctonus amoeruxi was identified through molecular cloning and it displayed moderate broad spectrum antimicrobial activities against representative strains of Gram-positive, Gram-negative bacteria and yeast in the range of 20-150 $\mu \mathrm{M}$ [24]. Named as AamAP1, the peptide is composed of 17 amino acids and displays a cationic ( +2 charge) $\alpha$-helical structure. In addition to the moderate antimicrobial activity reported for AamAP1, the peptide also displayed significant hemolytic activity against sheep erythrocytes at concentrations that were employed in the antimicrobial studies and showed no selectivity against mammalian cells.

In the present study, we aimed to employ the antimicrobial peptide AamAP1 as a platform for the design of a novel peptide analog with enhanced antibacterial activity and decreased cytolytic activity against eukaryotic cells based on enhancing the net positive charge on the parent peptide while optimizing other physico-chemical properties of the peptide that are known to influence AMPs activity. The strategy applied here produced a novel peptide named AamAP1-Lysine with enhanced antimicrobial activity and decreased toxicity towards mammalian cells. AamAP1-Lysine could prove to be a potential candidate for antimicrobial drug development in future studies.

\section{Experimental Section}

\subsection{Design of AamAP1-Lysine Based on Structural Determinants}

AamAP1-Lysine was designed based on increasing the net positive charge of the parent peptide while relatively optimizing other structural determinants that control AMPs activity. These structural determinants include: hydrophobicity $(\mathrm{H})$, hydrophobic moment $\left(\mathrm{M}_{\mathrm{H}}\right)$ and percentage helicity. Several peptide analogs were generated using this strategy and the peptide with the optimal structural determinants was selected (AamAP-1-Lysine) for further characterization of the effect of structural modification on the enhancement of peptide antimicrobial activity and target selectivity.

\subsection{Molecular Modeling and In Silico Analysis of AamAP1-Lysine}

The ProtParam software from the ExPASy server was employed for the evaluation of the physicochemical parameters of AamAP1-Lysine [25]. Structure prediction and the identification of the best template for homology modeling of AamAP1-Lysine were performed by the HHpred (HHsearch 2.0) software by HMM-HMM comparison [26]. The nearest template for reliable homology modeling was found with the RNA polymerase-associated protein Gp33 and showed a score of 20.9. Packing and solvent exposure characteristics were analyzed using the PROSA software [27]. Confirmation of the model reliability was performed using the I-TASSER software [28]. The RAMPAGE: Assessment of the Ramachandran Plot software was also used for the three dimensional structure validation [29]. The final model was visualized using Accelrys ${ }^{\circledR}$ Discovery studio software. 


\subsection{Peptide Synthesis and Purification}

AamAP1-Lysine was synthesized by the solid-phase method and Fmoc chemistry, purified by reverse phase high performance liquid chromatography using an acetonitirile $/ \mathrm{H}_{2} \mathrm{O}-\mathrm{TFA}$ gradient. The identity of AamAP1-Lysine was confirmed by ESI-MS mass spectrometry (GL Biochem Ltd., Shanghai, China).

\subsection{Bacterial Strains}

The bacterial strains used for the determination and testing of the antimicrobial activity of AamAP1 in this study were acquired from the American Type Culture Collection (ATCC, Manassas, VA, USA) and included: Staphylococcus auerus (ATCC 29213), Staphylococcus auerus (ATCC 43300), Staphylococcus auerus (ATCC 33591), Staphylococcus epidermidis (ATCC 12228), Enterococcus faecalis (ATCC 19433), Escherichia coli (ATCC 25922), Salmonella enterica (ATCC 10708), Pseudomonas aeruginosa (ATCC 9027), and Klebsiella pneumoniae (ATCC 13883).

\subsection{Bacterial Susceptibility Assay}

Susceptibility testing was performed by adopting the micro broth dilution method outlined by the Clinical and Laboratory Standards Institute (CLSI) guidelines using sterile 96-well plates [30,31]. Briefly, the organisms were removed from frozen glycerol stock and were grown overnight in Muller Hinton Broth $(\mathrm{MH})$ and diluted to $10^{6} \mathrm{CFU} / \mathrm{mL}$ in culture medium prior to use. Fifty $\mu \mathrm{L}$ of twofold serial dilutions of AamAP1-Lysine in the concentration range of 1-20 $\mu \mathrm{M}$ were added to $50 \mu \mathrm{L}$ bacteria in mid-log phase at a concentration of $10^{5} \mathrm{CFU} / \mathrm{mL}$ in 96 well microtitre plates. This was followed by incubating the plates for $18 \mathrm{~h}$, at $37{ }^{\circ} \mathrm{C}$, in a humidified atmosphere. Inhibition of microbial growth was determined by measuring the absorbance at $\lambda=570 \mathrm{~nm}$ with a microplate reader. Antibacterial activity was expressed as the minimal inhibitory concentration (MIC), for the minimum bactericidal concentration (MBC), $10 \mu \mathrm{L}$ of the well contents were spread on agar and grown at $37{ }^{\circ} \mathrm{C}$ for $24 \mathrm{~h}$ or $48 \mathrm{~h}$. The MBC was determined as the lowest concentration that resulted in $<0.1 \%$ survival of the subculture. All MIC and MBC determinations were made in triplicate.

\section{6. $\beta$-Galactosidase Assay}

The ability of the AamAP1-Lysine to inflict damage on the cytoplasmic membrane of bacterial cells and cause significant membrane permeabilization can be assessed by measuring the release of cytoplasmic $\beta$-galactosidase from E. coli (ML-35) cells into the culture medium using ONPG as substrate and as described previously [32]. E. coli cells were grown in LB medium $/ 5 \%$ lactose at $37{ }^{\circ} \mathrm{C}$ to an $\mathrm{OD}_{590 \mathrm{~nm}}$ of approximately 0.8 . The cells were later washed twice and centrifuged for $10 \mathrm{~min}$ at $1.4 \times g$ followed by resuspending the cells in PBS at the same OD. About $1 \times 10^{6}$ cells reconstituted in $100 \mu \mathrm{L}$ PBS and were incubated with different concentrations of AamAP1-Lysine. The hydrolysis of ONPG to O-nitro phenol by $\beta$-galactosidase enzyme was monitored at $405 \mathrm{~nm}$ using an ELISA plate reader over different time points $(10,20,30,40,50,60$ and $120 \mathrm{~min})$. 


\subsection{Extraction of Genomic DNA}

The Wizard $^{\circledR}$ Genomic DNA Purification Kit (Promega, Madison, WI, USA) was used for the isolation of $E$. coli bacterial genomic DNA according to the manufacturer instructions. The purity of the extracted DNA was considered of good quality if an optical ratio of DNA was in the range of OD260/OD280 $\geq 1.8$. A Thermo Scientific Nanodrop 1000 instrument (Wilmington, DE, USA) was used for determining the concentration of purified E. coli DNA at an absorbance of $260 \mathrm{~nm}$ and $280 \mathrm{~nm}$.

\subsection{DNA Gel Retardation}

The effect of AamAP1-Lysine on DNA gel retardation was preformed as described previously with minor modifications [33]. Briefly, increasing amounts of AamAP1-Lysine in $30 \mu \mathrm{L}$ binding buffer (10 mM Tris-HCl, $1 \mathrm{mM}$ EDTA buffer, $\mathrm{pH}$ 8.0) was mixed with $500 \mathrm{ng}$ of the genomic DNA. DNA migration was assessed by gel electrophoresis using a $0.8 \%$ agarose gel. The bands detected by fluorescence of eithidium bromide (EB). Finally gel retardation was visualized under UV illumination using a GelDoc-It ${ }^{\circledR}$ imaging system (UVP, Upland, CA, USA).

\subsection{Erythrocyte Hemolysis Assay}

The determination of the hemolytic activity of AamAP1-Lysine was performed as reported previously [34]. Briefly, a 4\% suspension of human erythrocytes was incubated with the peptide for 60 min. Centrifugation with $0.9 \% \mathrm{NaCl}$ was used for washing the human erythrocytes several times followed by incubation for $60 \mathrm{~min}$, at $37^{\circ} \mathrm{C}$. The cells were then centrifuged at $900 \times g$ for 5 min until the supernatant was separated from the pellet. Two hundred $\mu \mathrm{L}$ were transferred from each sample supernatant as 4 replicates into a 96-well plate and their absorbance measured at $570 \mathrm{~nm} .0 .9 \% \mathrm{NaCl}$ was used as the negative control, $0.1 \%$ Triton $\mathrm{X}-100$ as the positive control. The percent hemolysis was calculated using the following equation: hemolysis $=(\mathrm{A}-\mathrm{A} 0) /(\mathrm{AX}-\mathrm{A} 0) \times 100$, where $\mathrm{A}$ is OD $570 \mathrm{~nm}$ with the peptide solution, A0 is OD $570 \mathrm{~nm}$ in $\mathrm{NaCl}$, and $\mathrm{AX}$ is OD $570 \mathrm{~nm}$ with $0.1 \%$ Triton X-100.

\subsection{Cell Culture}

The following cell lines (HEK 293 Human Embryonic Kidney 293 cell line and Vero African green monkey kidney epithelial cell line), purchased from the ATCC were routinely cultured in RPMI 1640 or Dulbecco's modified Eagle's medium (DMEM) containing 10\% fetal bovine serum (FBS), 1\% L-glutamine, $1 \%$ sodium pyruvate, $50 \mathrm{U} / \mathrm{mL}$ penicillin and $50 \mathrm{mg} / \mathrm{mL}$ streptomycin. The cells were seeded into $150 \mathrm{~cm}^{2}$ culture flasks. Cells were cultured as monolayers in a humidified environment of $5 \% \mathrm{CO}_{2} 95 \%$ air at $37^{\circ} \mathrm{C}$.

\subsection{Cell Proliferation Assay}

The cytotoxicity of AamAP1-Lysine against cultured cells was determined by the MTT assay. Each cell line used in this experiment was seeded at a density of $5 \times 10^{3}$ cells per well into a 96-well microtitre plate containing $200 \mu \mathrm{L}$ serum free media. Cells were incubated with various concentrations 
of the peptide. After $24 \mathrm{~h}$ of incubation, $20 \mu \mathrm{L}$ of $5 \mathrm{mg} / \mathrm{mL}$ of 3-(4,5-dimethylthiazol-2-yl)-2,5diphenyltetrazolium bromide (MTT) was added and incubated for $4 \mathrm{~h}$. Conversion of MTT into purple formazan by metabolically active cells indicates the extent of cell viability. The medium was replaced by $200 \mu \mathrm{L}$ of dimethylsulfoxide (DMSO) and mixed to dissolve the formazan crystals that had developed. Absorbance was measured using an ELISA Microplate Reader at $550 \mathrm{~nm}$. The GraphPad Prism software was used for statistical analyses.

\section{Results}

\subsection{AamAP1-Lysine Design and Structural Modeling}

The peptide sequence of the natural scorpion venom antimicrobial peptide AamAP1 was used as a platform for the development of a novel synthetic peptide analog with enhanced antimicrobial activity. AamAP1 displays a net positive charge of +2 , percentage helicity of $66.6 \%$, a hydrophobic moment of 0.44 and a hydrophobicity average of 0.90 (Table 1). The rationale used for designing the peptide analog was to increase the positive charge on the parent peptide by substituting several amino acids found on the primary sequence with lysine amino acids while relatively optimizing other physico-chemical parameters of the parent peptide that are known to influence its activity such as hydrophobicity, hydrophobic moment, and helicity. As charge is considered a crucial structural determinant of antimicrobial activity as it is believed to be responsible for the initial interaction of cationic AMPs with the negatively charged bacterial membranes, an increase in the net positive charge is expected to enhance the antimicrobial activity of the parent peptide and minimize its toxicity. As shown in Table 1, five lysine amino acids were employed for substitution at positions 4, 8, 11, 12 and 16 on the parent peptide. The resultant peptide analog (AamAP1-Lysine) displays a net positive charge of +6 , percentage helicity of $88.3 \%$, a hydrophobic moment of 0.61 and a hydrophobicity average of 0.61 . The N-terminal amidation was unmodified due to several studies reporting loss of activity upon loss of amidation and the role on the N-terminal amidation in enhancing the biological activity of AMPs. Theoretical analysis of AamAP1-Lysine displayed that the peptide is adopting an alpha-helical structure when generated by the different methodologies mentioned earlier. The three dimensional model of AamAP1-Lysine revealed the peptide to exhibit an alpha helix conformation as generated by two different methodologies described previously (Figure 1). The Ramachandran plot was used for model validation and confirmed that $100 \%$ of the amino acids participating in forming the secondary structure of the peptide are in favored regions in relation to the phi and psi torsion angles of generated model. Additionally, a generated z-score of -1.78 was reported for AamAP1-Lysine using the PROSA II software which indicates that the generated model is of good quality.

Table 1. The amino acid sequences and properties of the peptides employed in this study.

\begin{tabular}{ccccc}
\hline Peptide & Sequence & Hydrophobicity (H) & $\begin{array}{c}\text { Hydrophobic } \\
\left.\text { moment } \mathbf{( M}_{\mathbf{H}}\right)\end{array}$ & Helicity \\
\hline AamAP1 & FLFSLIPHAIGGLISAFK & 0.90 & 0.44 & $66.6 \%$ \\
AamAP1-Lysine & FLFKLIPKAIKKLISKFK & 0.61 & 0.61 & $88.3 \%$ \\
\hline
\end{tabular}


Figure 1. Three-dimensional structural modeling of AamAp1-Lysine. Red regions correspond to helical structures within the peptide while the green regions represent hinged regions and unordered conformations, respectively. The structure was visualized using Accelrys Discovery studio software.

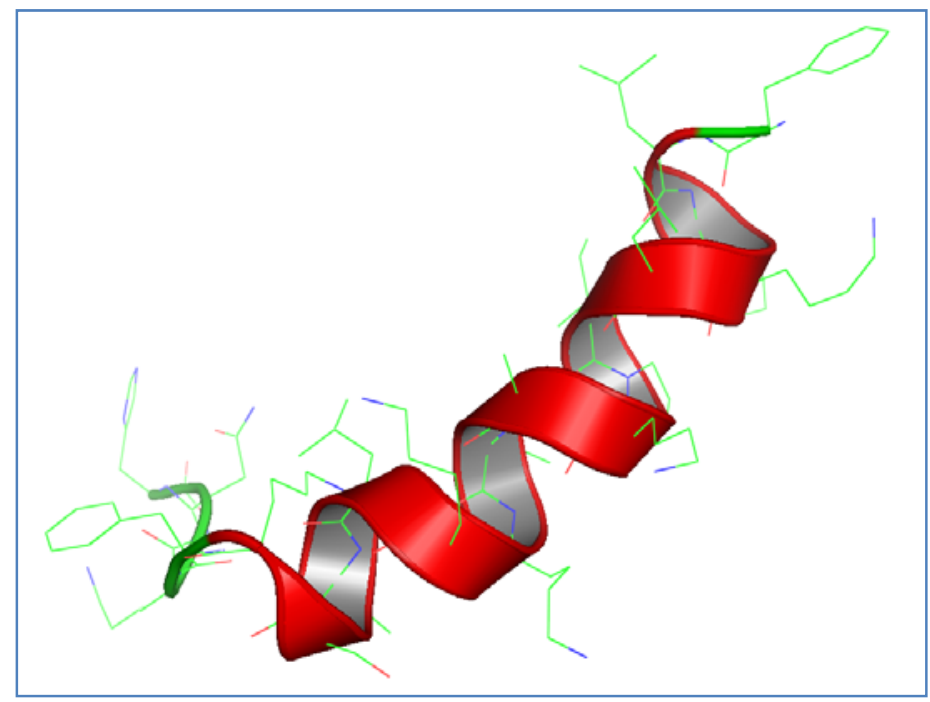

\subsection{Antimicrobial Activity of AamAP1-Lysine}

The broth micro-dilution method was employed for the investigation of the antimicrobial activity of AamAP1-Lysine against five strains of Gram-positive bacteria and four strains of Gram-negative bacteria. The MIC values of AamAP1-Lysine against the bacterial strains are summarized in Table 2. The modified antimicrobial peptide analog AamAP1-Lysine displayed potent antimicrobial activities against all bacterial strains studied with $S$. epidermidis, $S$. aureus (29213), S. aureus (43300), S. aureus (33591), E. faecalis, $P$. aeruginosa and K. pneumoniae being the most sensitive with MIC value of $5 \mu \mathrm{M}$. Additionally, the MIC value reported for AamAP1-Lysine against both Gram-negative strains of E. coli and S. enterica was $7.5 \mu \mathrm{M}$. The MBC values reported for AamAP1-Lysine against all bacterial strains studied were equal to the MIC values which indicate that the peptide analog is exhibiting a bactericidal antimicrobial nature.

Table 2. Minimum inhibitory concentrations (MICs) of AamAP1-Lysine against the test microorganisms employed in this study.

\begin{tabular}{ccc}
\hline Strain (Gram positive) & ATCC & MIC $(\boldsymbol{\mu M})$ \\
\hline Staphylococcus epidermidis & 12228 & 5 \\
Staphylococcus aureus & 29213 & 5 \\
Staphylococcus aureus & 43300 & 5 \\
Staphylococcus aureus & 33591 & 5 \\
Enterococcus faecalis & 19433 & 5 \\
\hline Strain(Gram negative) & ATCC & \\
\hline Eshereschia coli & 25922 & 7.5 \\
Salmonella enterica & 10708 & 7.5 \\
Pseudomonas aeruginosa & 9027 & 5 \\
Klebsiella pneumoniae & 13883 & 5 \\
\hline
\end{tabular}




\subsection{Hemolytic Activity of AamAP1-Lysine against Human Erythrocytes}

The hemolytic activity and potential toxicity of AamAP1-Lysine against human erythrocytes was examined at a concentration range of $1 \mu \mathrm{M}$ to $100 \mu \mathrm{M}$. Results of the hemolytic activity of AamAP1-Lysine are presented in Table 3. At concentrations of 5 and $10 \mu \mathrm{M}$, which corresponds to the MIC \& MBC concentration values reported for AamAP1-Lysine, the peptide induced minimal hemolysis of $(0 \%-1.38 \%)$ when incubated with human RBCs for sixty minutes. At a concentration of $80 \mu \mathrm{M}$, that is ten times greater than the geometric MIC value reported for the peptide against all tested bacterial strains, AamP1-Lysine induced a percentage hemolysis of $29.3 \%$. These results clearly indicate that AamP1-Lysine exhibits significant antimicrobial specificity and cell selectivity and weak hemolytic and RBC cytotoxicity.

Table 3. Hemolytic effect of AamAP1-Lysine on human erythrocytes after 60 min of incubation.

\begin{tabular}{cc}
\hline Peptide concentration $(\boldsymbol{\mu M})$ & Hemolysis $(\%)$ \\
\hline 1 & 0 \\
5 & 0 \\
10 & 1.38 \\
20 & 7.25 \\
40 & 16.58 \\
60 & 21.29 \\
80 & 29.32 \\
100 & 38.25 \\
\hline
\end{tabular}

\subsection{Viability of Eukaryotic Cells in Culture}

In addition to the hemolytic and cytolytic effects conducted with human erythrocytes, the antiproliferative activity of AamAP1-Lysine on the viability of two different eukaryotic cell lines (HEK 293 and Vero) was investigated and performed using the MTT assay. As shown in Figure 2, in the concentration range of AamAP1-Lysine needed to inhibit microbial growth (MIC of 5-10 $\mu \mathrm{M}$ ) against all bacterial strains employed in this study, the peptide exerted moderate cytotoxicity and the percentage cell viability was reported to be of $73 \%$ on average against both eukaryotic cell lines tested. At higher concentrations of $60-80 \mu \mathrm{M}$, a significant increase in cytotoxicity is observed with percentage cell viability varying from $55 \%$ to $42 \%$. These results suggest that application of AamAP1-Lysine at MIC concentrations can cause mild disruption of eukaryotic cells.

\subsection{Cytoplasmic Membrane Permeability}

To assess the effect of AamAP1-Lysine on the integrity of the cytoplasmic membrane of bacterial cells, the intracellular leakage and release of $\beta$-galactosidase from damaged bacterial cells degrades OPNG producing $o$-nitrophenol which can be monitored spectrophotometrically at $405 \mathrm{~nm}$. As shown in Figure 3, after treatment with different concentrations of AamAP1-Lysine, $\beta$-galactosidase was released from $E$. coli cells with a lag time of about $15 \mathrm{~min}$ and caused a steady increase in OD values over time which corresponds with $o$-nitrophenol formation. Results suggested that AamAP1-Lysine could permeabilize cytoplasmic membrane of $E$. coli cells in a dose dependent manner. 
Figure 2. Cell survival curves as measured by MTT assay for AamAP1-Lysine against two different eukaryotic cell lines HEK 293 and Vero cells lines. Cells were incubated with various concentrations of the peptide, for $24 \mathrm{~h}$, at $37{ }^{\circ} \mathrm{C}$. Control cells represent $100 \%$ proliferation, and the mean absorbance of treated cells was related to control values to determine sensitivity. Error bars represent standard error from mean cell proliferation as determined by repeated experiments.

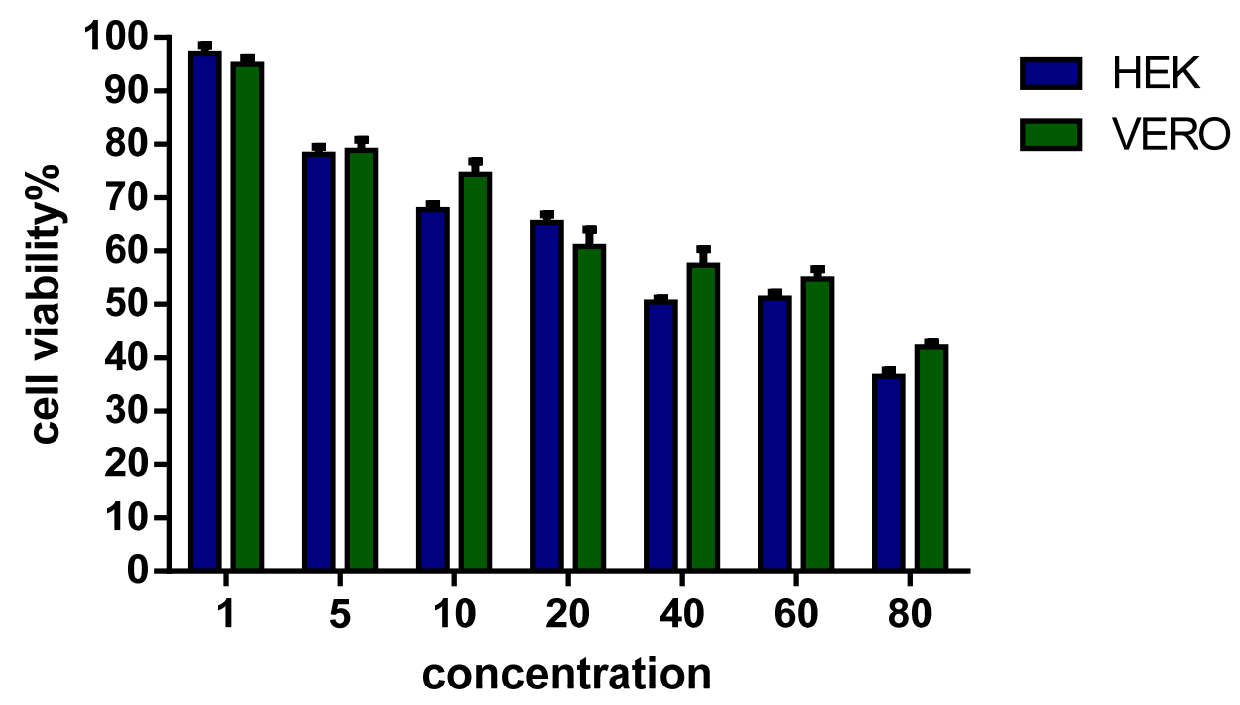

Figure 3. Release of cytoplasmic $\beta$-galactosidase from E. coli bacterial cells after treatment with four concentrations of AamAP1-Lysine $(7.5 \mu \mathrm{M}, 15 \mu \mathrm{M}, 22.5 \mu \mathrm{M}$ and $30 \mu \mathrm{M}$ ) or PBS (Negative control). The $\mathrm{Y}$ axis represents the optical density (OD) at $405 \mathrm{~nm}$. Data are representative of two independent experiments.

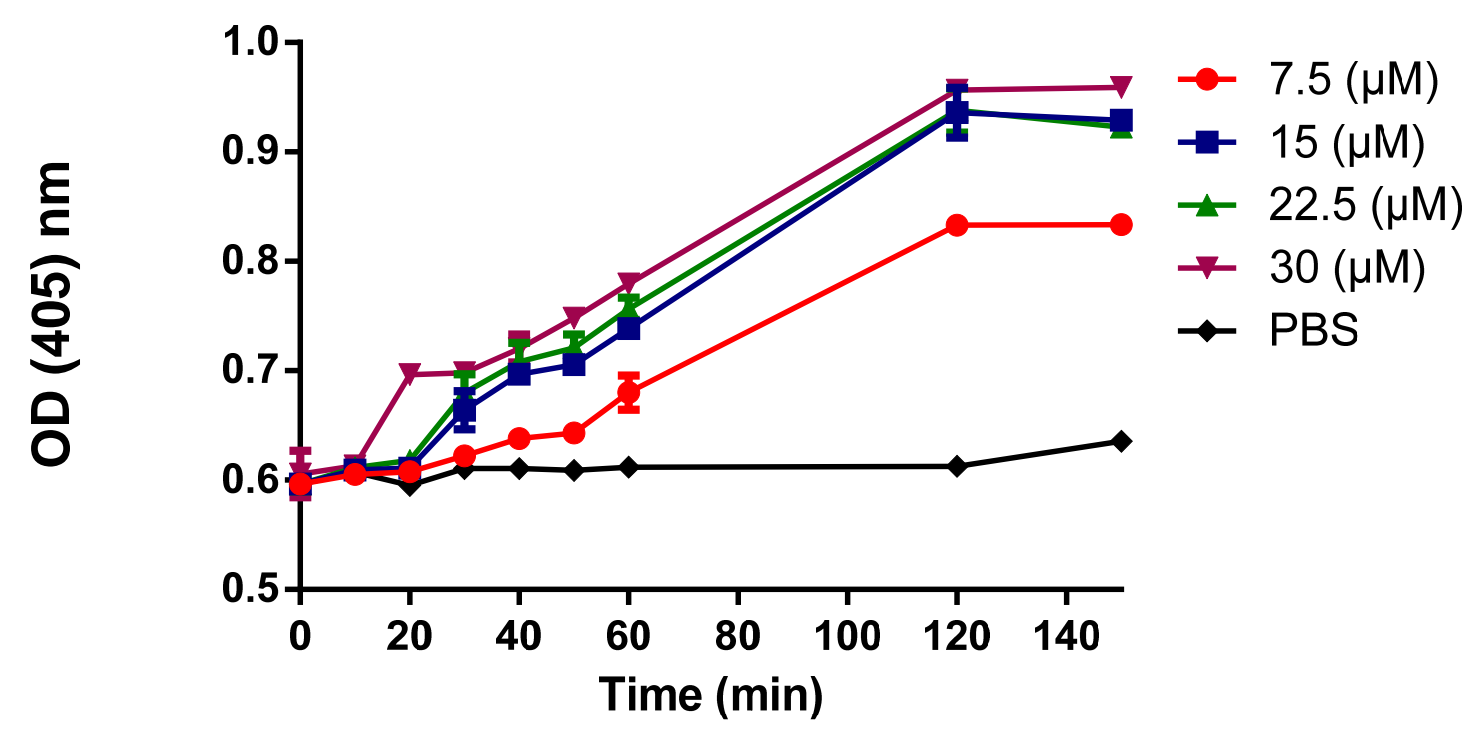

\subsection{DNA-Binding Activity}

The ability of AamAP1-Lysine to bind DNA and retard its migration was evaluated using an electrophoretic gel mobility shift assay. Different ratios of peptide to DNA were mixed with a fixed amount of E. coli genomic DNA, after which the mixture was electrophoresed on agarose gel. As shown in Figure 4. AamAP1-Lysine could interact with bacterial genomic DNA and cause a 
significant retardation in its movement starting from a peptide/DNA ratio of 1.9 which corresponds to $0.216 \mu \mathrm{g}$ of AamAP1-Lysine. At higher concentrations, the peptide also caused complete retardation of DNA migration indicating the peptide could possess intrinsic DNA binding abilities.

Figure 4. Gel retardation assays. Binding was assayed by the inhibitory effect of AamAP1-Lysine on the migration of DNA bands. Various amounts of peptides were incubated with $500 \mathrm{ng}$ of $E$. coli genomic DNA at room temperature for $10 \mathrm{~min}$, and the reaction mixtures were applied to a $0.8 \%$ agarose gel. The gel was visualized after ethidium bromide staining and UV irradiation. The numbers above the lanes represent the weight ratio (peptide/DNA).

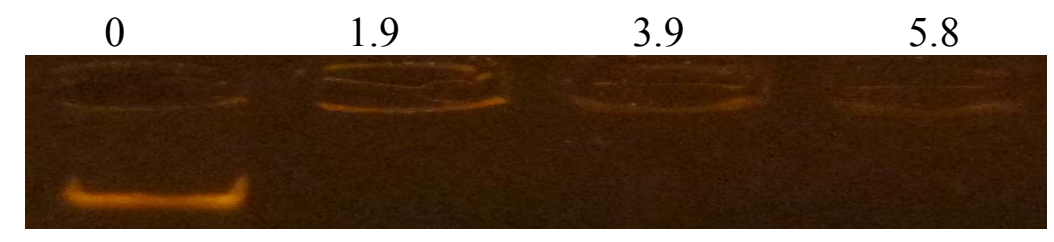

\section{Discussion}

Scorpion venoms contain a cocktail of bioactive peptides that display numerous biological activities [35]. Several scorpion venom peptides have been reported to display antimicrobial activities [35], and more than 40 scorpion venom antimicrobial peptides have been indentified and functionally characterized in the last decade [36]. The majority of these scorpion antimicrobial peptides display potent broad spectrum antimicrobial activity against representative strains of planktonic and multidrug resistant bacteria [36]. One of the major drawbacks of scorpion AMPs is the lack of evident microbial cell selectivity against target cells as the peptides induce strong hemolytic effects against mammalian cells and human erythrocytes [36]. The majority of scorpion AMPs are composed of 13-55 amino acids and display a cationic amphipathic alpha-helical structure and fit into the family of host defense peptides or AMPs in terms of activity and the characteristic structural determinants responsible for the mechanism of action and the molecular interaction between the peptide and target cellular membranes. AMPs have generated a great interest for their potential as drug candidates for drug development due to their potent antimicrobial activity and different mechanism of action when compared with conventional antibiotics. Several studies and an extensive amount of research have been conducted to elucidate the mechanism of action of AMPs and several theories have been provided including: the barrel stave model, the torroidal pore model and the carpet mechanism [37]. However, the exact mechanism of action of AMPs remains unknown and controversial till now. It is generally accepted that AMPs kill bacterial cells by mainly targeting bacterial membranes and inhibiting bacterial reproduction by binding microbial intracellular organelles [38,39]. Additionally, the activity of AMPs and cell selectively has been attributed to several structural determinants within the primary sequence of the peptide such as charge, helicity, hydrophobicity and hydrophobic moment [40]. Recently, a novel scorpion AMP has been identified through molecular cloning from the venom derived cDNA library of the North African scorpion Androctonus amoeruxi, named as AamAP1, the peptide displayed moderate antimicrobial activity in the range of $20-150 \mu \mathrm{M}$ against representative strains of Gram-positive and Gram-negative bacteria 
and suffered from significant toxicity against human erythrocytes [36]. In this project AamAP1 was used as a platform for the development of a new synthetic peptide analog with enhanced antimicrobial activity and decreased toxicity against mammalian cells. The synthetic analog, was modified to display an increase of +4 in the net positive charge while optimizing other structural determinants involved in the antimicrobial activity. Four amino acids on the parent peptide were substituted with lysine residues in order to increase the overall charge of the peptide. The resultant peptide analog, named as AamP1-Lysine displayed a net positive charge of +6 due to lysine substitution and exhibited a decrease in hydrophobicity from 0.90 to 0.61 and an increase in and hydrophobic moment from 0.44 to 0.61 respectively. Moreover, the amino acids substitution lead to an increase in percentage helicity from $66.3 \%$ to $83.3 \%$ as predicted by the NPS secondary structure prediction analyses (Swiss Model Workspace). The resultant changes in the structural parameters of AamAP1-Lysine lead to significant enhancement in the antimicrobial activity and cell selectivity of the peptide towards microbial cells. AamAP1-Lysine managed to inhibit bacterial growth for both Gram-positive and Gram-negative bacteria with an average geometric MIC of $7.5 \mu \mathrm{M}$ compared with the parent peptide which displayed an average MIC geometric of $85 \mu \mathrm{M}$. This 10 -fold increase in potency and antimicrobial activity could be explained by the optimized structural parameters AamAP1-Lysine possesses when compared to the parent peptide. Charge is considered crucial to the antimicrobial activity as it is responsible for the initial interaction of the antimicrobial peptides with the negatively charged phospholipids on microbial membranes [41]. Bacterial membranes are rich in the acidic phospholipids PG, PS, and CL which contributes collectively in conferring the overall negative charge on bacterial membranes [42]. Moreover, LPS and teichoic or teichuronic acids of Gram-negative and Gram-positive bacteria, impart additional negative charge to the surfaces of these respective organisms [42]. Thus, an increase in the cationic charge of AMPs could increase the electrostatic binding of the peptide to its target cell and consequently facilitate the killing mechanism. The increase in antimicrobial activity was also accompanied by a decrease in the hemolytic activity of the peptide against human erythrocytes. As reported earlier AamAP1-Lysine caused negligible hemolysis at MIC concentrations when compared with the parent peptide which was found to be strongly hemolytic and displayed weak selectivity towards microbial cells. The cytotoxicity of the peptide studies were also confirmed using the MTT cytotoxicity assay against human HEK 293 and Vero cell lines as the peptide caused mild disruption of eukaryotic cells. This improvement in the toxicity profile and selectivity index could also be attributed to the increase in cationic charge and improved binding to microbial membranes. Other factors could have also contributed to the improvement of the selectivity index as the helicity of the peptide and hydrophobicity that were increased due to structural modifications on the parent peptide and are considered as major contributors of effective antimicrobial activity and target selectivity [43]. To confirm the helicity and the adoption of an $\alpha$-helical structure by AamAp1-Lysine, the secondary structure of the peptide was constructed using in silico homology modeling and the model generated clearly displays a stable alpha helical structure in confirmation with our preliminary analysis results.

Finally and in order to confirm the membrane targeted mechanism of action of AamAP1-Lysine, the membrane permeabilization ability of the peptide in addition to its ability to bind genomic bacterial DNA was investigated. AamP1-Lysine managed to increase $o$-nitrophenol formation as indicated by the increase in disruption of the membrane's integrity due to leakage of intracellular $\beta$-galactosidase in a dose dependent manner. Moreover the peptide was able to cause complete retardation of the 
migration of bacterial genomic DNA at all peptide concentrations employed. This could indicate that the peptide could display dual mechanisms by killing bacteria due to membrane damage in addition to the binding of genomic DNA.

\section{Conclusions}

In summary, we report the design and functional characterization of the antimicrobial and cytotoxic activity of a synthetic scorpion venom peptide analog with enhanced potent antibacterial activities and low hemolytic and cytotoxic activities against eukaryotic cells. Several additional studies are needed to assess the activity and potential toxicity of AamAP1-Lysine using in vivo animal models but the preliminary results obtained from this study indicate that AamAP1-Lysine could have the potential for the development into an effective novel antimicrobial agent.

\section{Conflicts of Interest}

The authors declare no conflict of interest.

\section{References}

1. Alanis, A.J. Resistance to antibiotics: Are we in the post-antibiotic era? Arch. Med. Res. 2005, 36, 697-705.

2. Heymann, D.L. Resistance to anti-infective drugs and the threat to public health. Cell 2006, 124, 671-675.

3. Paphitou, N.I. Antimicrobial resistance: action to combat the rising microbial challenges. Int. J. Antimicrob. Agents 2013, 42, S25-S28.

4. Hawkey, P.M.; Jones, A.M. The changing epidemiology of resistance. J. Antimicrob. Chemother. 2009, 64 (Suppl. 1), i3-i10.

5. Rice, L.B. The clinical consequences of antimicrobial resistance. Curr. Opin. Microbiol. 2009, 12, 476-481.

6. Spellberg, B.; Guidos, R.; Gilbert, D.; Bradley, J.; Boucher, H.W.; Scheld, W.M.; Bartlett, J.G.; Edwards, J., Jr. the Infectious Diseases Society of America. The epidemic of antibiotic-resistant infections: A call to action for the medical community from the Infectious Diseases Society of America. Clin. Infect. Dis. 2008, 46, 155-164.

7. Spellberg, B.; Powers, J.H.; Brass, E.P.; Miller, L.G.; Edwards, J.E., Jr. Trends in antimicrobial drug development: Implications for the future. Clin. Infect. Dis. 2004, 38, 1279-1286.

8. Chen, Y.; Vasil, A.I.; Rehaume, L.; Mant, C.T.; Burns, J.L.; Vasil, M.L.; Hancock, R.E.W.; Hodges, R.S. Comparison of biophysical and biologic properties of $\alpha$-helical enantiomeric antimicrobial peptides. Chem. Boil. Drug Des. 2006, 67, 162-173.

9. Chen, Y.; Guarnieri, M.T.; Vasil, A.I.; Vasil, M.L.; Mant, C.T.; Hodges, R.S. Role of peptide hydrophobicity in the mechanism of action of $\alpha$-helical antimicrobial peptides. Antimicrob. Agents Chemother. 2007, 51, 1398-1406. 
10. Kraus, D.; Peschel, A. Molecular mechanisms of bacterial resistance to antimicrobial peptides. In Antimicrobial Peptides and Human Disease; Springer: Berlin/Heidelberg, Germany, 2006; pp. 231-250.

11. Zasloff, M. Antimicrobial peptides of multicellular organisms. Nature 2002, 415, 389-395.

12. Jenssen, H.; Hamill, P.; Hancock, R.E.W. Peptide antimicrobial agents. Clin. Microbial. Rev. 2006, 19, 491-511.

13. Nicolas, P.; Mor, A. Peptides as weapons against microorganisms in the chemical defense system of vertebrates. Annu. Rev. Microbiol. 1995, 49, 277-304.

14. Nguyen, L.T.; Haney, E.F.; Vogel, H.J. The expanding scope of antimicrobial peptide structures and their modes of action. Trends Biotechnol. 2011, 29, 464-472.

15. Wiradharma, N.; Khoe, U.; Hauser, C.A.E.; Seow, S.V.; Zhang, S.; Yanga, Y.-Y. Synthetic cationic amphiphilic $\alpha$-helical peptides as antimicrobial agents. Biomaterials 2011, 32, 2204-2212.

16. Stark, M.; Liu, L.P.; Deber, C.M. Cationic hydrophobic peptides with antimicrobial activity. Antimicrob. Agents Chemother. 2002, 46, 3585-3590.

17. Lee, E.K.; Kim, Y.C.; Nan, Y.H.; Shin, S.Y. Cell selectivity, mechanism of action and LPS-neutralizing activity of bovine myeloid antimicrobial peptide-18 (BMAP-18) and its analogs. Peptides 2011, 32, 1123-1130.

18. Li, L.; Shi, Y.H.; Cheserek, M.J.; Su, G.F.; Le, G.W. Antibacterial activity and dual mechanisms of peptide analog derived from cell-penetrating peptide against Salmonella typhimurium and Streptococcus pyogenes. Appl. Microbiol. Biotechnol. 2013, 97, 1711-1723.

19. Zhao, Z.; Ma, Y.; Dai, C.; Zhao, R.; Li, S.R.; Wu, Y.; Cao, Z.; Li, W. Imcroporin, a new cationic antimicrobial peptide from the venom of the scorpion Isometrus maculates. Antimicrob. Agents Chemother. 2009, 53, 3472-3477.

20. Yuan, W.; Cao, L.; Ma, Y.; Mao, P.; Wang, W.; Zhao, R.; Wu, Y.; Cao, Z.; Li, W. Cloning and functional characterization of a new antimicrobial peptide gene StCT1 from the venom of the scorpion Scorpiops tibetanus. Peptides 2010, 31, 22-26.

21. Silva, É.C.N.; Camargos, T.S.; Maranhão, A.Q.; Silva-Pereira, L.; Silva, L.P.; Possani, L.D.; Schwartz, E.F. Cloning and characterization of cDNA sequences encoding for new venom peptides of the Brazilian scorpion Opisthacanthus cayaporum. Toxicon 2009, 54, 252-261.

22. Luna-Ramírez, K.; Quintero-Hernández, V.; Vargas-Jaimes, L.; Batista, C.V.F.; Winkel, K.D.; Possani, L.D. Characterization of the venom from the Australian scorpion Urodacus yaschenkoi: Molecular mass analysis of components, cDNA sequences and peptides with antimicrobial activity. Toxicon 2013, 63, 44-54.

23. Torres-Larios, A.; Gurrola, G.B.; Zamudio, F.Z.; Possani, L.D. Hadrurin, a new antimicrobial peptide from the venom of the scorpion Hadrurus aztecus. Eur. J. Biochem. 2000, 267, 5023-5031.

24. Almaaytah, A.; Zhou, M.; Wang, L.; Chen, T.; Walker, B.; Shaw, C. Antimicrobial/cytolytic peptides from the venom of the North African scorpion, Androctonus amoreuxi: Biochemical and functional characterization of natural peptides and a single site-substituted analog. Peptides 2012, 35, 291-299.

25. Gasteiger, E.; Gattiker, A.; Hoogland, C.; Ivanyi, I.; Appel, R.D.; Bairoch, A. ExPASy: The proteomics server for in-depth protein knowledge and analysis. Nucleic Acids Res. 2003, 31, 3784-3788. 
26. Söding, J.; Biegert, A.; Lupas, A.N. The HHpred interactive server for protein homology detection and structure prediction. Nucleic Acids Res. 2005, 33 (Suppl. 2), W244-W248.

27. Wiederstein, M.; Sippl, M.J. ProSA-web: Interactive web service for the recognition of errors in three-dimensional structures of proteins. Nucleic Acids Res. 2007, 35 (Suppl. 2), W407-W410.

28. Zhang, Y. I-TASSER server for protein 3D structure prediction. BMC Bioinform. 2008, 9, doi:10.1186/1471-2105-9-40.

29. Šali, A.; Blundell, T.L. Comparative protein modelling by satisfaction of spatial restraints. J. Mol. Biol. 1993, 234, 779-815.

30. Komatsuzawa, H.; Ohtaa, K.; Sugaia, M.; Fujiwaraa, T.; Glanzmannb, P.; Berger-Bächib, B.; Suginaka, H. Tn551-mediated insertional inactivation of the fmtB gene encoding a cell wall-associated protein abolishes methicillin resistance in Staphylococcus aureus. J. Antimicrob. Chemother. 2000, 45, 421-431.

31. Ouhara, K.; Komatsuzawa, K.; Kawai, T.; Nishi, H.; Fujiwara, T.; Fujiue, Y.; Kuwabara, M.; Sayama, K.; Hashimoto, K.; Sugai, M. Increased resistance to cationic antimicrobial peptide LL-37 in methicillin-resistant strains of Staphylococcus aureus. J. Antimicrob. Chemother. 2008, $61,1266-1269$.

32. Je, J.Y.; Kim, S.K. Chitosan derivatives killed bacteria by disrupting the outer and inner membrane. J. Agric. Food Chem. 2006, 54, 6629-6633.

33. Imura, Y.; Nishida, M; Ogawa, Y.; Takakura, Y.; Matsuzaki, K. Action mechanism of tachyplesin I and effects of PEGylation. Biochim. Biophys. Acta (BBA)-Biomembr. 2007, 1768, 1160-1169.

34. Almaaytah, A.; Tarazi, S.; Mhaidat, N.; Al-Balas, Q.; Mukattash, T.L. Mauriporin, a novel cationic $\alpha$-helical peptide with selective cytotoxic activity against prostate cancer cell lines from the venom of the scorpion Androctonus mauritanicus. Int. J. Pept. Res. Ther. 2013, 19, 281-293.

35. Hmed, B.N.; Serria, H.T.; Mounir, Z.K. Scorpion peptides: Potential use for new drug development. J. Toxicol. 2013, 2013, 958797.

36. Almaaytah, A.; Albalas, O. Scorpion venom peptides with no disulfide bridges: A review. Peptides 2014, 51, 35-45.

37. Wimley, W.C.; Hristova, K. Antimicrobial peptides: Successes, challenges and unanswered questions. J. Membr. Biol. 2011, 239, 27-34.

38. Brogden, N.K.; Brogden, K.A. Will new generations of modified antimicrobial peptides improve their potential as pharmaceuticals? Int. J. Antimicrob. Agents 2011, 38, 217-225.

39. Tagai, C.; Morita, S.; Shiraishi, T.; Miyaji, K.; Iwamuro, S. Antimicrobial properties of arginine-and lysine-rich histones and involvement of bacterial outer membrane protease $\mathrm{T}$ in their differential mode of actions. Peptides 2011, 32, 2003-2009.

40. Huang, Y.; Huang, J.; Chen, Y. Alpha-helical cationic antimicrobial peptides: Relationships of structure and function. Protein Cell 2010, 1, 143-152.

41. Findlay, B.; Zhanel, G.G.; Schweizer, F. Cationic amphiphiles, a new generation of antimicrobials inspired by the natural antimicrobial peptide scaffold. Antimicrobi. Agents Chemother 2010, 54, 4049-4058.

42. Yeaman, M.R.; Nannette, Y.Y. Mechanisms of antimicrobial peptide action and resistance. Pharmacol. Rev. 2003, 55, 27-55. 
43. Takahashi, D.; Sanjeev, K.S; Om, P.; Guolong, Z. Structural determinants of host defense peptides for antimicrobial activity and target cell selectivity. Biochimie. 2010, 92, 1236-1241.

(C) 2014 by the authors; licensee MDPI, Basel, Switzerland. This article is an open access article distributed under the terms and conditions of the Creative Commons Attribution license (http://creativecommons.org/licenses/by/3.0/). 